\title{
32 Stoma
}

\author{
Ekkehard C. Jehle
}

\subsection{Kapitelzusammenfassung}

Ein nicht unerheblicher Anteil der Patienten mit koloproktologischen Krankheitsbildern benötigt vorübergehend oder auch auf Dauer ein Stoma. Stomaanlagen erfordern in gleichem Maße wie alle anderen koloproktologischen Operationen eine hohe chirurgische Sorgfalt. Entscheidend für die Akzeptanz eines Stomas durch den Patienten sind eine gute präoperative Aufklärung und Vorbereitung, die Indikationsstellung zum „richtigen“ Stoma, die technisch perfekte Anlage an der richtigen Stelle und eine intensive postoperative Anleitung und Edukation durch engagierte Stomatherapeuten. Im Zusammenspiel all dieser Maßnahmen kann der Stoma-Patient - vorübergehend oder auch dauerhaft - eine sehr gute Lebensqualität erreichen.

\subsection{Definitionen}

\subsubsection{Begrifflichkeiten}

Das Wort Stoma kommt aus dem Griechischen und bedeutet Mund, Öffnung. Der Plural heißt dementsprechend Stomata. Im medizinischen (chirurgischen) Sprachgebrauch wird der Begriff Stoma für verschiedene Situationen benützt, zum einen für die Bezeichnung von Anastomosen, also neugeschaffene Verbindungen zwischen Hohlorganen (z. B. Gastroenterostomie oder Ileoascendostomie), zum anderen für neugeschaffene Abflussöffnungen des harnableitenden Systems (Urostoma) oder des Darmtrakts (Enterostoma). Der korrespondierende Ausdruck im Englischen ist „ostomy“. Während Chirurgen von einem Kolostoma oder Ileostoma sprechen, wird von den Enterotherapeuten meist die Bezeichnung Kolostomie, Ileostomie oder auch Urostomie benützt. Bei den Patienten und in der Laienpresse sind Begriffe wie „künstlicher Darmausgang“, „Seitausgang“ oder auch „Kunstafter“ gebräuchlich.

Das folgende Kapitel beschäftigt sich ausschließlich mit den Enterostomata.

\subsubsection{Anatomische Beschreibung}

Im üblichen chirurgischen Sprachgebrauch wird ein Enterostoma mit der anatomischen Position bezeichnet, also Ileostoma, Jejunostoma, Kolostoma, oder noch exakter Transversostoma, Descendendostoma oder Sigmoidostoma. 


\subsubsection{Stomaarten}

Man unterscheidet weiterhin zwischen endständigen Stomata (engl. „terminal“) und doppelläufigen Stomata. Für die doppelläufigen Stomata gibt es eine Fülle von Synonymen: Loop-Ileostoma, Deviationsstoma, protektives Stoma, im Englischen „diverting ostomy“ oder „defunctioning ostomy“. Endständige Stomata können vorübergehend („temporär“) oder aber definitiv angelegt werden (siehe 32.3.1). Doppelläufige Stomata werden außer in wenigen Ausnahmefällen nicht als definitive Lösung, sondern in temporärer Intention angelegt (siehe 32.3.2).

Eine Mischform zwischen doppelläufigem und endständigem Stoma stellt ein Split-Stoma dar, es vereinigt die Vorteile beider Stomaarten: Es ist technisch leicht anzulegen und vor allem auch wieder rückzuverlegen, ähnlich wie ein doppelläufiges Stoma. Es schaltet - anders als ein doppelläufiges und wie ein „normales“ endständiges Stoma - die Stuhlpassage jedoch komplett aus.

\subsection{Indikationen zur Stomaanlage}

\subsubsection{Temporäres Stoma}

Temporäre Stomata werden angelegt:

- Um eine Anastomose, bei der die Gefahr einer Anastomosen-Insuffizienz als hoch eingeschätzt wird, vor der Stuhlpassage zu schützen. Die Anlage eines Stomas verhindert dabei nicht die Anastomoseninsuffizienz, sondern verhindert im besten Fall die Folgen dieser Insuffizienz, eben z. B. eine Peritonitis oder einen Beckenabszess. Typische temporäre Stomata für diesen Zweck sind ein LoopIleostoma oder auch ein doppelläufiges Transversostoma bei einer anterioren Rektumresektion mit koloanaler Anastomose oder ein Loop-Ileostoma bei Proktokolektomie mit ileopouchanaler Anastomose.

- Wenn der Operateur das Risiko einer Anastomose als zu hoch einschätzt. Typisches Beispiel hierfür ist die Diskontinuitätsresektion nach Hartmann bei der perforierten Sigmadivertikulitis mit Peritonitis.

- Im Vorgriff auf eine definitive Lösung oder um eine solche überhaupt durchführen zu können. Typisches Beispiel hierfür ist die Anlage eines Loop-Ileostoma bei stenosierendem Rektumkarzinom in einem kurativen Therapiekonzept vor neoadjuvanter Radiochemotherapie und daran anschließender Operation.

- Bei Crohn-Patienten mit analem Fistelleiden zur Inaktivierung der Fisteln vor plastischer Rekonstruktion.

- Bei aufwändigen plastischen Rekonstruktionen im Sphinkter-/Beckenbodenbereich. 


\subsubsection{Permanentes (definitives) Stoma}

Permanente Stomata werden angelegt, wenn eine andere Lösung mit Erhalt der natürlichen Stuhlpassage nicht möglich oder nicht sinnvoll ist. Typische Beispiele hierfür sind:

- Ein endständiges Kolostoma bei Patienten mit Rektumkarzinom und Infiltration des Sphinkters und somit Indikation zur Rektumexstirpation.

- Ein endständiges Kolostoma bei Patienten mit Rektumkarzinom und vorbestehender Einschränkung der Stuhlkontinenz.

- Ein endständiges Kolostoma bei Patienten mit Stuhlinkontinenz und Einschränkung der Lebensqualität ohne die Option einer operativen Korrektur.

- Ein endständiges Ileostoma bei Patienten nach Proktokolektomie bei therapierefraktärer Colitis Crohn und analem Fisteleiden.

- Ein doppelläufiges Jejuno- oder Ileostoma bei Peritonealkarzinose und Ileus.

\subsection{Vorbereitung des Patienten}

Selbst wenn bei vielen Patienten ein Stoma nur temporär für wenige Wochen besteht und selbst wenn bei vielen Patienten mit einer geplanten definitiven Stomaanlage die Lebensqualität anschließend deutlich verbessert sein wird, bedeutet eine Stomaanlage einen erheblichen Einschnitt in die körperliche Integrität und das Körpergefühl eines Menschen. Deshalb muss dies gut vorbereitet werden.

\subsubsection{Prästationäre Aufklärung}

Bei geplanten Stomaanlagen muss dem Patienten genügend Zeit gegeben werden, sich mit diesem Thema zu beschäftigen. Anfänglich wird der Vorschlag des Chirurgen zur Stomaanlage, selbst dann, wenn der Patient sich mit dem Thema schon beschäftigt hat, auf Zurückhaltung und Widerstände treffen. Sinnvoll ist es deshalb, die Patienten von entsprechenden Patienten-Organisationen (ILCO, DCCV), noch besser direkt von „erfahrenen Stomaträgern“ gleichen Geschlechts und ähnlichen Alters, die dem Chirurgen persönlich bekannt sind, beraten zu lassen. Im eigenen Vorgehen werden den Patienten in der Sprechstunde Telefon-Nummern von ehemaligen eigenen Patienten, die Stomaträger sind und sich zu dieser Beratung bereit erklärt haben, ausgehändigt. Zusätzlich wird schon in der Sprechstunde der erste Kontakt mit der eigenen Stomatherapeutin hergestellt. Den Patienten wird zudem ein selbst verfasster Stomaratgeber ausgehändigt. Eine Aufklärung der Patienten für eine geplante Stomaanlage erst am Tag vor der Operation sollte - außer bei Notfällen oder dringlichen OPs - vermieden werden. 


\subsubsection{Präoperative Maßnahmen}

\section{Chirurgische Aufklårung}

Die chirurgische Aufklärung über eine Stomaanlage sollte nicht nur dann erfolgen, wenn eine Stomaanlage geplant und zwingend erforderlich ist, sondern auch dann, wenn im Rahmen einer komplexen Operation oder auf Grund der präoperativen Diagnostik ein Risiko für eine Stomaanlage besteht. Insbesondere gilt dies bei komplexen Operationen bei Morbus Crohn, bei Peritonealkarzinose und bei multidisziplinären Eingriffen (siehe Abschnitt Multidisziplinäre Operationen). Diese Aufklärung sollte immer vor dem Kontakt mit dem Stomatherapeuten erfolgen.

\section{Aufklärung durch Stomatherapeuten}

Die Aufklärung durch den Stomatherapeuten beinhaltet Informationen über die postoperative Versorgung des Stomas, die postoperative Anleitung zur Selbstversorgung, die anschließende Überleitung in den ambulanten Bereich und den Alltag als Stomaträger. Zusätzlich werden konkrete Fragen der Patienten beantwortet.

Integraler Bestandteil der präoperativen Betreuung durch den Stomatherapeuten ist das Probekleben der Stomaversorgung. Dabei müssen die patientenspezifischen Voraussetzungen und auch Wünsche bezüglich der Positionierung des Stomas berücksichtigt werden. Entscheidend für eine spätere gute Versorgbarkeit des Stomas ist - neben der perfekten chirurgischen Anlage - die richtige Position an der Bauchdecke. Prinzipiell soll ein Stoma durch den Musculus rectus abdominis ausgeleitet werden und nicht lateral durch die schräge Bauchmuskulatur. Es müssen Falten und Narben nach Voroperationen, aber auch der Verlauf eines Hosengürtels oder RockBundes berücksichtigt werden. Neben der Überprüfung der „richtigen“ Positionierung im Stehen und Liegen ist aber die richtige Position im Sitzen entscheidend: Hier erst werden Bauchfalten erst richtig erkennbar. Für eine gute Versorgbarkeit ist eine „glatte“ Bauchwandstelle von mindestens $4 \mathrm{~cm}$ Durchmesser erforderlich. Zudem muss bei adipösen Patienten überprüft werden, ob dieser die Stomastelle im Stehen und Sitzen ohne Hilfe eines Spiegels sehen kann, damit er das Stoma später eigenständig versorgen kann. Falls nicht, muss gegebenenfalls das Stoma auch weit im Oberbauch angezeichnet werden. Für die richtige Positionierung und Suche der optimalen Position muss sich der Stomatherapeut ausreichend Zeit nehmen, da eine „falsche“ Position erhebliche Auswirkungen auf die Lebensqualität des Patienten hat.

\section{Anzeichnen}

Ist die optimale Position gefunden, wird diese mit nicht abwaschbarem (Edding-) Stift an der Bauchdecke markiert. Im eigenen Vorgehen werden prinzipiell mindestens zwei Stomastellen, eine links und eine rechts angezeichnet, damit der Operateur - falls nötig - zwei Optionen für die Anlage hat. Meist ist das Anzeichnen der Stoma-Position einfach. Im Zweifelsfall - vor allem bei vielen Voroperationen und 
schon früheren Stomaanlagen - muss für das Stoma gemeinsam von Stomatherapeut und Operateur die richtige Lokalisation gefunden werden; in komplexen Situationen bietet es sich an, mehrere mögliche Stomapositionen anzuzeichnen.

Auch in der Notfallsituation sollte noch präoperativ die Stomaposition angezeichnet werden, die Zeit reicht dafür immer aus; auch ein Notfallpatient lässt sich im OP kurzzeitig zum Anzeichnen noch in eine sitzende Position aufrichten. Falls dies nicht möglich ist oder sich erst während der Operation die nicht antizipierte Notwendigkeit einer Stomaanlage ergibt, empfiehlt es sich, bei schlanken Patienten das Stoma in Nabelhöhe, bei adipösen Patienten im Oberbauch anzulegen.

\section{Dokumentation}

Die angezeichneten Stomastellen samt ebenfalls angezeichneten Falten und Narben werden im eigenen Vorgehen fotodokumentiert und im KIS bei den Patienten-Dokumenten abgespeichert, sodass, falls die Markierungen nicht mehr sichtbar sein sollten, sich diese im OP leicht rekonstruieren lassen (Abb. 32.1).

\section{Lavage}

Soll operativ lediglich ein Loop-Ileostoma ohne Darmresektion (z. B. zur Inaktivierung von Analfisteln oder vor neoadjuvanter RCTx bei stenosierendem Rektumkarzinom) angelegt werden, empfiehlt sich dennoch die Darmlavage, da ansonsten durch das verbleibende stuhlgefüllte Colon keine komplette Inaktivierung stattfindet und sich zudem der ,alte“ Stuhl im Colon eindickt. Falls dies präoperativ nicht stattgefunden hat, sollte postoperativ eine Spülung des Colons über den abführenden Schenkel des Stomas erfolgen.
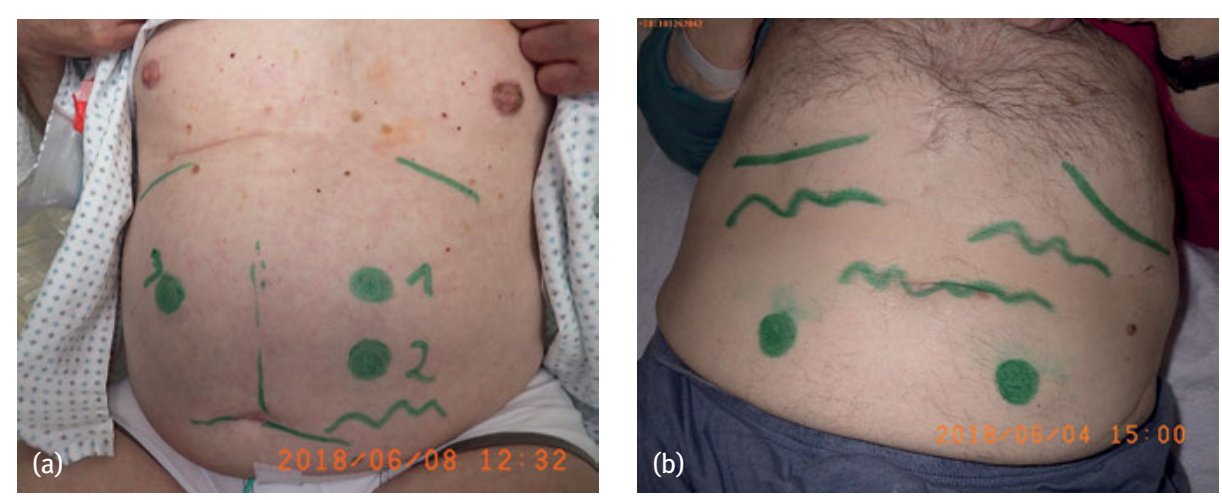

Abb. 32.1: Präoperativ angezeichnete und dokumentierte Stomapositionen. (a) (links) Angezeichnet sind Narben, eine Hautfalte, die Rippenbögen und drei mögliche Stomapositionen. (b) (rechts) Angezeichnet sind die Rippenbögen, drei Hautfalten und zwei Stomapositionen im Unterbauch. 


\section{Multidisziplinåre Operationen}

Zunehmend werden Visceralchirurgen bei Tumoroperationen anderer operativer Disziplinen, insbesondere in der Gynäkologie bei Patientinnen mit Ovarialkarzinom involviert. Auch hier müssen die o.g. Abläufe eingehalten werden. Im eigenen interdisziplinären Vorgehen werden alle diese Tumorpatientinnen prinzipiell präoperativ koloskopiert, vom Chirurgen rektoskopiert, es erfolgt eine Darmvorbereitung, die Patientinnen werden vom Chirurgen über potentiell notwendige Darmoperationen und Stomaanlagen aufgeklärt, die Stomatherapeutin zeichnet Stomapunkte an. Damit wird verhindert, dass die Patientin und der Chirurg in eine nicht antizipierte und deshalb technisch nicht perfekt lösbare Situation kommen. Analog gilt dies für Patienten mit fortgeschrittenen urologischen Tumoren.

\subsection{OP-Technik}

\subsubsection{Prinzipien}

Stomata sollen prinzipiell (an der vorher angezeichneten Stelle) durch einen „Tunnel“ durch die Rektusscheide hindurch angelegt werden (Abb.32.2). Die vordere Rektusscheide, die Rektusmuskulatur und - außer im Unterbauch - die hintere Rektusscheide verhindern besser eine parastomale Hernie als ein Durchzug durch die schräge Bauchmuskulatur. Nach Exzision der Haut wird das subkutane Fettgewebe nicht ausgeschnitten, sondern nur zur Seite gedrängt; die vordere Rektusscheide wird kreuzförmig inzidiert, die Rektusmuskulatur wird nicht reseziert, sondern ebenfalls nur zur Seite gedrängt; die hintere Rektusscheide und das Peritoneum (oder im Unterbauch kaudal der Linea arcuata nur das Peritoneum) werden stumpf perforiert und gegebenenfalls zusätzlich inzidiert. Die Durchtrittsstelle soll gerade so weit sein, dass der Darm mit seinem Mesenterium und dem zu antizipierenden Stuhlgang im Lumen

(a)

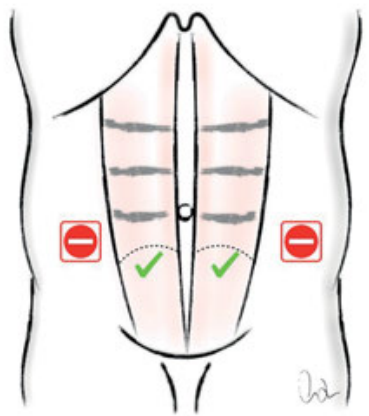

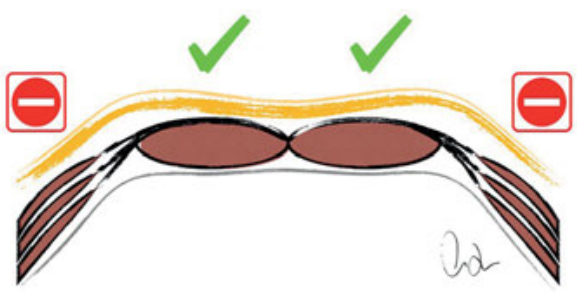

(b)

Abb. 32.2: Schematische Darstellung der Stomadurchtrittstellen durch die Bauchwand (mit freundlicher Genehmigung von Prof. Dr. C. Isbert, Hamburg). (a) Frontalansicht. (b) Querschnitt durch die Bauchdecke. 
nicht eingeengt ist, andererseits nicht zu weit, damit das Risiko einer parastomalen Hernie minimiert wird. Das Stoma muss gut durchblutet, ohne Spannung und vom Lumen her mit dem Zeigefinger bis durch die Bauchdecke hindurch durchgängig sein. Falls diese Kriterien nicht erfüllt sein sollten, muss - auch nach Bauchdeckenverschluss oder Entfernung der Trokare beim laparoskopischen Vorgehen - das Stoma neu angelegt werden. Der durchgezogene Darm wird im eigenen Vorgehen innen an der Durchtrittsstelle nicht fixiert.

Bei adipösen Patienten muss - insbesondere bei Anlage eines endständigen Kolostomas im Unterbauch - die erheblich größere benötigte Länge im Sitzen antizipiert werden (und gegebenenfalls der Darm innen entsprechend mobilisiert werden), da sonst schon unmittelbar postoperativ bei der Mobilisation des Patienten eine Stomaretraktion oder ein Stomaausriss droht.

\subsubsection{Ileostoma/Jejunostoma}

Dünndarm-Stomata müssen prinzipiell anders angelegt werden als Kolostomata: Da der Dünndarmstuhl durch die darin enthaltenen Gallensäuren sehr aggressiv ist und der dünnflüssige Dünndarm-Stuhl auch leicht die Stomaplatte „unterwandern“ kann, muss ein Dünndarmstoma stets prominent mit einem „Nippel“ angelegt werden, damit die Stomaplatte millimetergenau zugeschnitten und somit die parastomale Haut optimal geschützt werden kann.

\section{Loop-Ileostoma}

Ein prominentes Loop-Ileostoma lässt sich leicht durch asymmetrisches Eröffnen des Darmes und Umstülpen des zuführenden Schenkels erreichen; der zuführende Schenkel wird dann in dieser Form mittels „Dreipunktnaht“ als „Nippel“, der abführende Schenkel plan in die Haut eingenäht. Auch ein prominentes Einnähen sowohl von zuführendem als auch abführendem Schenkel ist möglich. Im eigenen Vorgehen wird für die Anlage ein „Reiter“ oder „Stoma-Steg“ benützt (Abb. 32.3). Dieser erleichtert das nippelförmige Anlegen. Dieser Steg kann aus einem industriell angefertigten oder aber auch aus einem selbst konstruierten Gummizügel bestehen. Ein Stoma darf natürlich - auch mit einem Reiter - nie unter Spannung angelegt werden.

\section{Endstån diges Ileostoma}

Ein endständiges Ileostoma wird ebenfalls durch Umstülpen des Darmes und nippelförmiges Einnähen durch Dreipunkt-Naht angelegt. Im eigenen Vorgehen kommt das Mesenterium kranial zu liegen und dient als Hypomochlion (Abb. 32.4).

Wenn die Anlage eines endständigen Ileostomas mit Umstülpen des Darmes aus Gründen fehlender Länge des Mesenteriums oder wegen der Dysproportion zwischen Durchmesser des Darmes und des Mesos schwierig ist, kann es technisch einfacher 

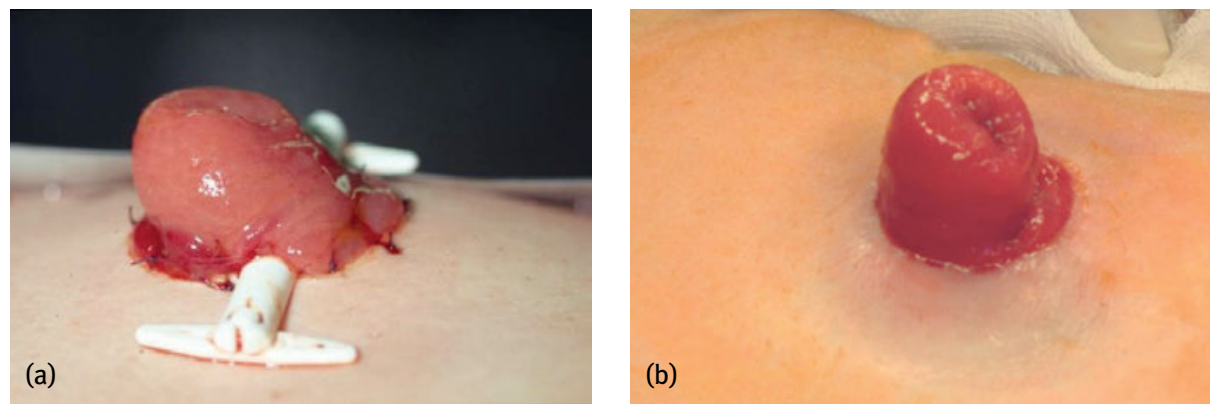

Abb. 32.3: Loop-Ileostoma mit prominent eingenähtem zuführendem Schenkel und plan in die Bauchdecke eingenähtem abführenden Schenkel. (a) Intraoperativ mit „Reiter“. (b) Eingeheiltes Loop-lleostoma.

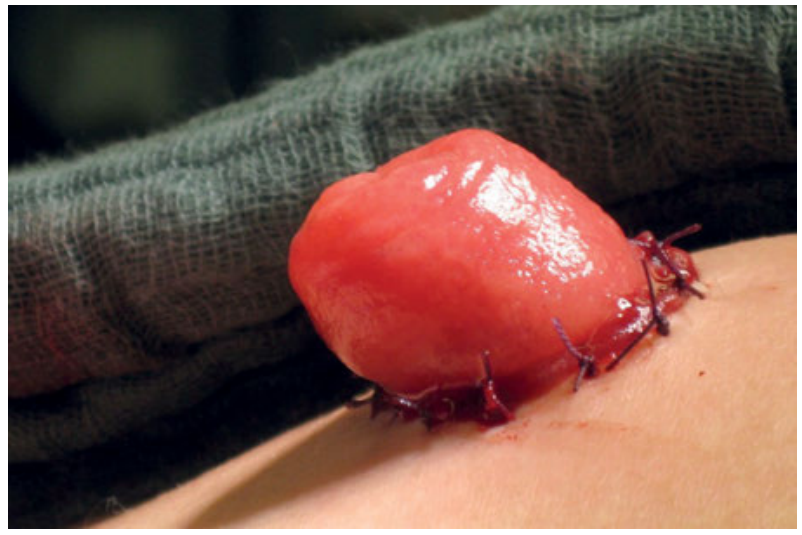

Abb. 32.4: Endständiges nippelförmiges Ileostoma.

sein, das endständige Ileostoma in der Technik eines Loop-Ileostomas in „Krückstock-Form“ anzulegen. Falls auch dies nicht möglich ist, kann ein Dünndarmstoma als „Chimney“ ausgeleitet werden: d.h. der Darm wird kaminartig lediglich vor die Bauchdecke gezogen, dort mit Nähten fixiert und eröffnet, das Meso bleibt somit frei und unbedeckt. In den ersten Wochen erfordert solch ein Chimney erhöhte stomatherapeutische Zuwendung, nach einigen Wochen sieht dieses jedoch wie ein „normales“ Ileostoma aus, die Mucosa ist über das Mesenterium gewachsen.

\subsubsection{Kolostoma}

\section{Doppellåufiges Kolostoma}

Die Häufigkeit der Anlage eines protektiven Kolostomas hat in den letzten Jahren deutlich und zu Gunsten der Ileostomaanlage abgenommen. Die Vorteile eines protektiven Kolostomas sind die geringere Stomafördermenge und der weniger aggres- 
sive Dickdarm-Stuhlgang mit somit einem geringeren Risiko von Hautirritationen. Der Nachteil der doppelläufigen Kolostomata ist ein regelhaft auftretender Prolaps, sodass sich das ,atompilzartige“ Stoma auch mit großen Stomaplatten durch den Patienten nur mit Mühe versorgen lässt.

Technisch ist die Stoma-Anlage einfach, sie wird meist im rechten oder linken Colon transversum oder im Colon sigmoideum durchgeführt und sollte natürlich spannungsfrei sein. Im eigenen Vorgehen wird das Stoma über einem Reiter ca. $1 \mathrm{~cm}$ prominent eingenäht.

\section{Endståndiges Kolostoma}

Endständige Kolostomata werden entweder in temporärer Intention im Rahmen einer Diskontinuitätsresektion (Hartmann-OP) oder aber als permanentes Stoma angelegt, dies typischerweise bei einer Rektumexstirpation oder bei gynäkologischen und urologischen Tumoren des Beckens in einem palliativen Therapiekonzept.

Kolostomata sollen „ein wenig“ prominent, also ca. $1 \mathrm{~cm}$ über Bauchdeckenniveau angelegt werden, um gut versorgbar zu sein. Da endständige Kolostomata meist im linken Mittel- oder Unterbauch angelegt werden, muss die zusätzlich benötigte Darmlänge in sitzender Position bei der Anlage antizipiert werden (siehe auch Abschnitt Probekleben). Bei definitiven Kolostomata besteht die Option einer späteren Irrigation. Dies ermöglicht dem Patienten zusätzliche Lebensqualität (siehe auch Kap. 32.7.2 Ambulante Stomatherapie, Home Care Unternehmen, Irrigation). Um die Irrigation zu erleichtern, sollte eine intraabdominelle Schleifenbildung des Kolons vermieden werden, eventuell muss hierfür noch Kolon nachreseziert werden. Im eigenen Vorgehen werden permanente Kolostomata immer so angelegt, dass später eine Irrigation möglich ist.

\subsection{Stomakomplikationen}

\subsubsection{Frühe Komplikationen}

Frühe Stomakomplikationen können entweder durch objektive patientenspezifische Schwierigkeiten, aber auch durch mangelnde chirurgische Sorgfalt bedingt sein. Sollte schon während oder am Ende der Operation erkennbar sein, dass das angelegte Stoma nicht die erforderliche Qualität hat, muss alles versucht werden, dies intraoperativ noch zu korrigieren: Die unten angeführten Komplikationen können den postoperativen Verlauf deutlich erschweren und verlängern, im Extremfall das Überleben des Patienten gefährden, zumindest jedoch die Lebensqualität des Patienten deutlich einschränken. 


\section{Retraktion}

Eine früh im postoperativen Verlauf auftretende Stomaretraktion kann durch den Operations-Situs bedingt sein, z. B. bei ausgeprägt adipösen Bauchdecken oder fehlender Mobilisierbarkeit des Darmes bei Peritonealkarzinose. Häufig ist die Ursache jedoch die nicht ausreichende Mobilisierung des Darmes, weil die erforderliche Länge für eine spannungsfreie Anlage nicht richtig antizipiert wurde. Dies betrifft insbesondere die Stomaanlage bei adipösen Patienten im Unterbauch, bei denen im Sitzen häufig zusätzlich über 10 Zentimeter Strecke mehr von Nöten sind (siehe hierzu auch Abschnitt Probekleben).

Bei nur geringfügiger Retraktion und ansonsten vitalem Darm lässt sich die Situation bei einem Kolostoma meist mit stomatherapeutischen Maßnahmen beherrschen. Bei stärkerer Retraktion und bei einem Ileostoma muss fast immer eine Revisionsoperation erfolgen.

\section{Nekrose}

Eine früh-postoperative Stoma-Nekrose ist häufig mit einer Stoma-Retraktion verbunden. Es kann sich jedoch auch um ein eigenständiges Problem, bedingt durch eine zu enge Tunnelierung durch die Bauchdecke oder eine $z u$ ausgeprägte Abpräparation des Mesos handeln. Oberflächliche Schleimhautnekrosen, unter denen sich eine vitale Darmwand befindet, benötigen keine chirurgische Therapie. Ausgeprägtere Nekrosen bedürfen meist einer operativen Revision.

\section{Mukokutane Separation}

Eine mukokutane Separation ist die häufigste und harmloseste Form einer Stomanekrose oder Stomaretraktion, meist bedingt durch kleinere Durchblutungsstörungen an der Stomakante oder auch eine zu groß gewählte Hautexzision. Mukokutane Separationen bedürfen intensiverer stomatherapeutischer Maßnahmen, lassen sich darunter aber immer zur Abheilung bringen.

\section{Obstruktion}

Eine Stomaobstruktion ist eine sehr häufige postoperative Komplikation, insbesondere bei Ileostomata. Sie ist meist bedingt durch ein - regelhaft auftretendes - StomaÖdem und der dadurch verursachten relativen Stenose im Bauchdeckenniveau, kann aber auch durch eine Knickstenose des Dünndarmes ausgelöst werden. Fast immer lässt sich die Stuhlpassage durch Intubation des Stomas mit einem Blasenkatheter, die orale Gabe von Laxantien oder auch eine intravenöse Darmstimulation mit Neostigmin herstellen. Falls die vorgenannten Maßnahmen nicht erfolgreich sein sollten, kann eine retrograde „therapeutische“ Dünndarmdarstellung über das Ileostoma bis zum Duodenum die Passage regelhaft wiederherstellen (Abb.32.5). Operative Maßnahmen sind nicht notwendig. 


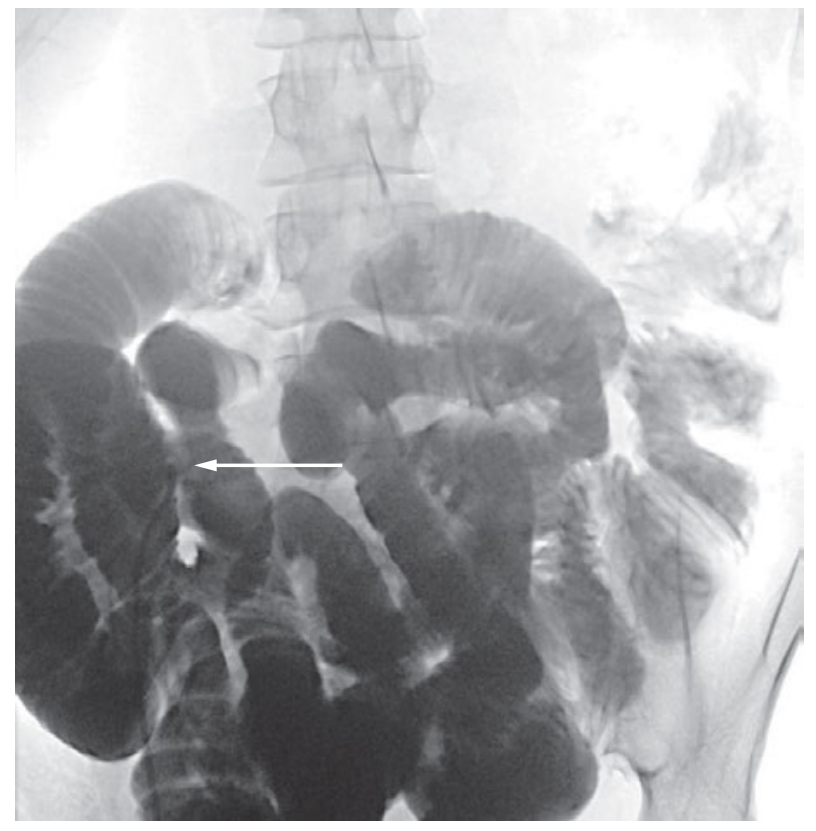

Abb. 32.5: Therapeutische retrograde Darstellung des Dünndarmes über das endständige Ileostoma bei postoperativer Obstruktions-Symptomatik nach Kolektomie. Stoma mit einliegendem Katheter mit Pfeil markiert.

\section{High Output Stoma (HOS)}

Ein „High Output Stoma“ (HOS) tritt fast ausschließlich bei Dünndarm-Stomata auf, insbesondere dann, wenn längere Strecken Dünndarm entfernt werden mussten (anatomisches Kurzdarm-Syndrom) oder zur Stoma-Anlage aus technischen Gründen ein oberer Dünndarmanteil benutzt werden musste (funktioneller Kurzdarm), es kann aber auch bei normalen Ileostomata mit ausreichender Dünndarmlänge auftreten. Auch bei Kolostomata ist ein HOS in Ausnahmefällen möglich.

Eine verbindliche Definition der Menge des Flüssigkeitsverlustes bei einem HOS gibt es nicht. In der eigenen Definition liegt ein HOS dann vor, wenn der Patient durch alleiniges Trinken den Flüssigkeitsverlust nicht ausgleichen kann und auf eine zusätzliche intravenöse Flüssigkeitsgabe angewiesen ist. In der eigenen Praxis wird eine Stomafördermenge von maximal $1000 \mathrm{ml}$ pro Tag angestrebt. Dann benötigt der Stomaträger sicher keine zusätzliche Flüssigkeits-Substitution.

Ein HOS tritt sehr häufig als Frühkomplikation nach Stomaanlage auf. Es sollte unter stationären Bedingungen sofort erkannt und therapiert werden. Bei Nichterkennen oder ohne adäquate Therapie ist das HOS lebensbedrohend, vor allem bei geriatrischen Patienten.

Entscheidend ist die tägliche Bilanzierung der Stomafördermenge. Bei einem Stoma-Output von mehr als $1000 \mathrm{ml}$ benötigt der Patient eine parenterale Flüssigkeitszufuhr und stuhlmodulierende Maßnahmen. Einfache therapeutische Maßnahmen sind eindickende Nahrung und die Gabe von Quellmitteln (Plantago ovata). Im eigenen Vorgehen ist der nächste Schritt die Gabe von Tinctura opii in langsam steigen- 
der Dosierung. Nach erreichter Höchstdosis und noch nicht ausreichender Wirkung kann dies zusätzlich mit Loperamid kombiniert werden. In Ausnahmefällen kann die zusätzliche Applikation von H2-Blockern und Octreotid notwendig werden. Eine Entlassung eines Patienten mit einem bestehenden HOS aus der stationären Behandlung sollte möglichst nicht oder wenn, dann nur nach Organisation der Weiterbetreuung durch den Hausarzt erfolgen.

\subsubsection{Späte Komplikationen}

\section{Parastomale Hernie}

Parastomale Hernien treten sehr häufig auf. Bei temporären Stomata spielen sie klinisch keine Rolle. Bei definitiven Stomata stellen sie nur dann die Indikation zu einer operativen Korrektur dar, wenn das Stoma sich nicht mehr ordentlich versorgen lässt oder eine rezidivierende Obstruktionssymptomatik auftritt. Gelegentlich kann auch die eingeschränkte Kosmetik eine OP-Indikation darstellen. Für die operative Korrektur gibt es verschiedene Techniken: Das Stoma kann an anderer Stelle neu angelegt, es kann von außen eine Stomakorrektur, meist mit Mesh-Augmentation, oder auch - heute am häufigsten durchgeführt - eine laparoskopische Korrektur mit MeshEinlage durchgeführt werden.

Da eine so hohe Inzidenz parastomaler Hernien besteht, wird zunehmend die Netzverstärkung der Stomadurchtrittsstelle schon bei der primären Stoma-Anlage propagiert. Dieses Vorgehen beinhaltet jedoch Risiken vor allem bezüglich Infektionen, sodass im Moment die primäre Netzverstärkung zur Prävention der parastomalen Hernie nicht als Standard empfohlen werden kann.

\section{Retraktion}

Eine sekundäre Stomaretraktion ist fast ausschließlich die Folge einer Gewichtszunahme der Patienten. Diese müssen dementsprechend aufgeklärt werden und versuchen, wieder abzunehmen.

\section{Stomaprolaps}

Ein Stomaprolaps tritt fast ausschließlich bei endständigen Kolostomata auf. Ein kleiner Prolaps braucht nicht korrigiert zu werden; größere Prolapse, insbesondere mit rezidivierenden Durchblutungsstörungen, sollten operiert werde. Auch hier kommen äußere Verfahren mit der Resektion des prolabierenden Darmes und Neu-Einnähung des Stomas wie auch laparoskopische Techniken zum Einsatz. Letzteres bietet sich an, wenn zusätzlich eine parastomale Hernie besteht. 


\section{High output Stoma (HOS)}

Bei einigen Patienten, insbesondere bei solchen mit einem anatomischen oder funktionellen Kurzdarm vor einem Ileo- oder Jejunostoma, kann auch über längere Zeit ein High output Stoma bestehen. Bei diesen Patienten muss zusätzlich zur Bilanzierung und Modulation der Stomafördermenge und der Flüssigkeitssubstitution (siehe auch Abschnitt HOS) eine Substitution von Kalorien, Spurenelementen und Vitaminen erfolgen. Hier ist die Anbindung an eine spezialisierte gastroenterologische Institution sinnvoll.

\subsection{Patientenedukation und -anleitung}

\subsubsection{Postoperative stationäre Betreuung}

Wie schon oben dargestellt, erfolgte im Idealfall die Aufklärung und die Auseinandersetzung des Patienten mit der Stomaanlage schon im Vorfeld in der Sprechstunde und im Kontakt mit Stomatherapeutin, Selbsthilfegruppen und „erfahrenen“ Stomaträgern (siehe auch Kap. 32.4.1 Prästationäre Aufklärung). Präoperativ erfolgt dann auf der Station das Probekleben und das Anzeichnen der optimalen Stomalokalisation (siehe hierzu auch Aufklärung durch Stomatherapeuten und Probekleben).

\section{Anleitung zur Versorgung}

Postoperativ wir der Patient täglich von der Stomatherapeutin gesehen, vom Pflegepersonal wird täglich die Stomafördermenge bilanziert, vom Chirurgen werden Maßnahmen zur Stuhlmodulation verordnet (siehe Abschnitt HOS). Der Patient und gegebenenfalls auch dessen Angehörige werden in die Stomaversorgung eingelernt und zu eigenständigem Leeren des Stomabeutels und eigenständigem Zuschneiden der Platte angeleitet. Hierzu wird dem Patienten von der Stomatherapeutin eine Schablone angefertigt und ausgehändigt. Zum Zeitpunkt der Entlassung soll der Patient in der Lage sein, eigenständig und ohne fremde Hilfe (außer der seiner Angehörigen) mit der Stomaversorgung zurecht zu kommen.

\section{Edukation}

Durch den Stomatherapeuten und den Chirurgen wird auch eine Patientenedukation durchgeführt. Die Patienten werden dabei über potentielle Stomaprobleme aufgeklärt, insbesondere in Hinblick auf ein High output Stoma: Bilanzierung, Früherkennung und therapeutische Maßnahmen. Im eigenen Vorgehen gibt es ergänzend hierzu selbstentwickelte Flyer zur Ernährung bei Stomapatienten und zu Maßnahmen bei High output Stoma, welche dem Patienten ausgehändigt werden. 


\section{Dokumentation, Ůbergabe}

Alle Maßnahmen im Rahmen der Stomatherapie, -anleitung und -edukation sollten dokumentiert werden. Es erfolgt dann eine strukturierte Übergabe in den ambulanten Bereich mit einem Übergabeprotokoll. Die für den täglichen Gebrauch benötigten Stoma-Artikel sollten schon vorher bestellt und damit zuhause verfügbar sein, damit keine Versorgungslücke auftritt.

\subsubsection{Ambulante Stomatherapie, Home Care Unternehmen, Irrigation}

Die Weiterbetreuung der Stomapatienten durch ein ambulantes Home Care Unternehmen bereitet zunehmend Probleme, da die Versorgung mit Stoma-Artikeln mittlerweile budgetiert ist und einige Krankenkassen Flächenverträge mit nur einem einzigen Home Care Unternehmen abgeschlossen haben. Dies erfordert umso mehr, dass die Patienten aus den Kliniken optimal geschult und informiert entlassen werden.

Eine wichtige Aufgabe der ambulanten Stomatherapie ist die Anleitung zur Irrigation bei permanenten Kolostomata. Dies kann, da nach der morgendlichen mit einem geringen Zeitaufwand durchgeführten Irrigation das Stoma lediglich mit einer „Stomakappe“ mit Gasfilter abgedeckt wird und kein Stomabeutel getragen werden muss, erheblich zur Lebensqualität des Patienten beitragen (Abb. 32.6).

(a)
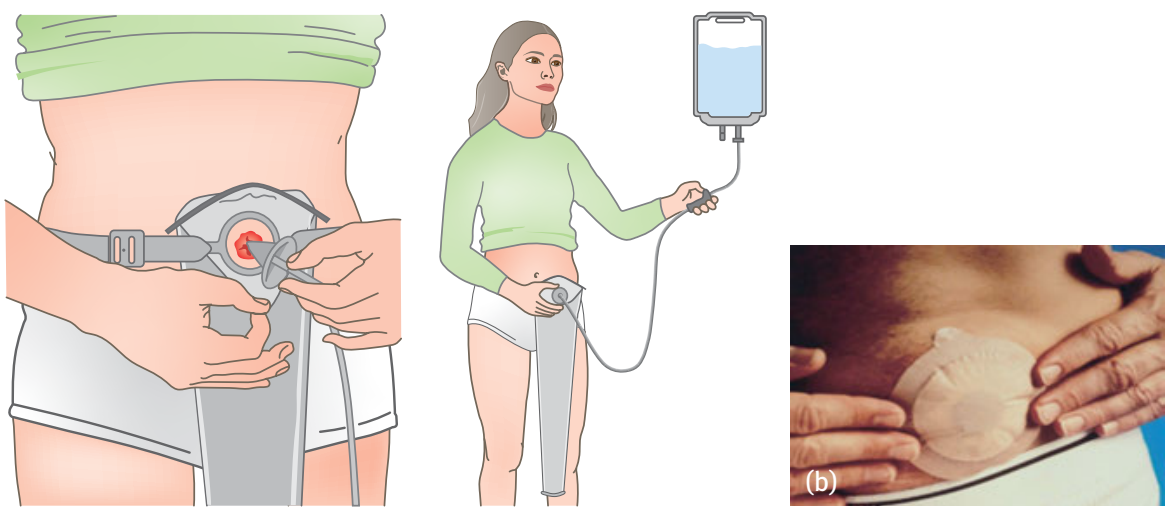

Abb. 32.6: Stoma-Irrigation. (a) Schematische Darstellung der Irrigationsbehandlung bei einem Kolostoma. (b) Kolostoma-Versorgung nach Irrigation mit „Stomakappe“. 


\subsection{Stomaresektion - Stomarückverlagerung - Re-Anastomosierung}

\subsubsection{Timing der Stomarückverlagerung oder Re-Anastomosierung}

Am häufigsten werden Loop-Ileostomata oder auch doppelläufige Kolostomata bei Patienten mit tiefer anteriorer Rektumresektion wegen eines Rektumkarzinoms angelegt. Wenn bei diesen eine neoadjuvante Radiochemotherapie durchgeführt wurde und postoperativ die adjuvante Chemotherapie (CTx) geplant ist, sollte das Timing der Stoma-Rückverlagerung zusammen mit den betreuenden Onkologen festgelegt werden. Bei infusionaler 5-FU-Gabe kann die Stomaresektion zwischen den einzelnen CTx-Zyklen oder aber auch nach Beendigung der 4 postoperativen CTx-Zyklen erfolgen; bei der heute üblichen oralen Capecitabine-Einnahme wird die Stomaresektion üblicherweise erst nach Beendigung der CTx durchgeführt. Prinzipiell ist bei ungestörter Anastomosenabheilung auch eine sehr frühe Rückverlagerung 7-10 Tage nach der Erst-OP möglich.

Die Ileostomaresektion nach Pouch-OP erfolgt im eigenen Vorgehen 6 Wochen postoperativ, in den meisten anderen Kliniken nach 3 Monaten.

Eine Re-Anastomosierung erfolgt meist nach einer Diskontinuitäts-Resektion, welche wegen einer Perforation mit Peritonitis oder wegen einer Anastomoseninsuffizienz notwendig war. Der richtige Zeitpunkt für die Re-Anastomosierung hängt somit vom ursprünglichen Ausmaß der Peritonitis, gegebenenfalls auch von der onkologischen Situation ab. Eine Re-Anastomosierung sollte in dieser Situation frühestens nach 3 Monaten erfolgen.

\subsubsection{Rückverlagerung Kolostoma, Resektion Ileostoma, Anastomosierung Split-Stoma}

Voraussetzung für die Rückverlagerung eines Stomas, welches in protektiver Intention angelegt wurde, ist der Nachweis, dass die zu schützende Anastomose intakt oder auch der plastische Fistelverschluss erfolgreich war. Es wird deshalb im eigenen Vorgehen prinzipiell vom Chirurgen eine proktologische Untersuchung mit Rektoskopie oder Pouchoskopie sowie vom Radiologen eine Defäkographie mit flüssigem jodhaltigem Kontrastmittel (Ultravist) durchgeführt. Die Defäkographie ist die sensitivste Methode zum Nachweis von Leckagen oder Fisteln. Bei einem doppelläufigen Kolostoma kann bei der Rückverlagerung im Regelfall ein einfacher Nahtverschluss durchgeführt werden, da die Lumina so weit sind, dass keine Stenosierung auftritt. Bei Loop-Ileostomata muss jedoch im Regelfall, da der abführende Schenkel häufig auf ein sehr kleines Lumen geschrumpft ist, eine Resektion mit Anastomosierung erfolgen. Im eigenen Vorgehen wird eine weite Seit-zu-Seit- Klammernaht-Anastomose 
angelegt. Bei einem Split-Stoma wird ebenfalls typischerweise eine Seit-zu-Seit-Anastomose angelegt.

\subsubsection{Re-Anastomosierung}

Die Re-Anastomosierung nach einer Diskontinuitätsresektion stellt einen ganz anderen Schweregrad des Eingriffs dar und erfordert eine Re-Laparotomie oder eine ReLaparoskopie.

Die häufigste durchgeführte Reanastomosierung erfolgt nach einer Hartmann-OP bei perforierter Sigmadivertikulitis. Dies stellt wegen der vorhergehenden Peritonitis häufig eine komplexe Operation mit ausgedehnter Adhäsiolyse und Mobilisierung der linken Kolonflexur. dar. Es muss betont werden, dass gerade bei älteren und multimorbiden Patienten aus diesem Grund häufig keine Re-Anastomosierung durchgeführt wird. Technisch wird die Anastomose meist ohne ausgedehnte Freipräparation des Rektumstumpfes durch einfaches „Durch-Stapeln“ mit dem Rund-KlammernahtGerät durchgeführt.

\section{Weiterführende Literatur}

Chudner A, Gachabayov M, Dyatlov A, Lee H, Essani R, Bergamaschi R. The influence of diverting loop ileostomy vs. colostomy on postoperative morbidity in restorative anterior resection for rectal cancer: a systematic review and meta-analysis. Langenbecks Arch Surg. 2019;doi: 10.1007/s00423-019-01758-1.

Danielsen AK, Burcharth J, Rosenberg J. Patient education has a positive effect in patients with a stoma: a systematic review. Colorectal Dis. 2013;15:276-283.

Gavriilidis P, Azoulay D, Taflampas P. Transverse colostomy versus loop ileostomy for defunctioning of colorectal anastomosis: a systematic review, updated conventional meta-analysis, and cumulative meta-analysis. Surg Today. 2019;49:108-117.

Justiniano CF, Temple LK, Swanger AA, et al. Readmissions with dehydration after ileostomy creation: Rethinking risk factors. Dis Colon Rectum. 2018;61:1297-1305.

Keighley MRB. Stomas an related problems. In: Keighley MRB, Williams NS (eds.). Surgery of the anus, rectum and colon. W. B. Saunders, London. 1993, 139-294.

Sailer M. Stomatherapie und Stomakomplikationen. In: Sailer M, Aigner F, Hetzer FH (eds.). Expertise Koloproktologie. 1. Auflage. Thieme, Stuttgart. 2016, 348-355.

Seo YJ, Bailey K, Aguayo E, Juo YY, et al. Readmissions after ileostomy creation using a Nationwide Database. Am Surg. 2018;84:1661-1664. 